\title{
The association between smoking and breast cancer characteristics and outcome
}

\author{
Hadar Goldvaser ${ }^{1,2^{*}}$, Omer Gal ${ }^{1}$, Shulamith Rizel ${ }^{1,2}$, Daniel Hendler ${ }^{1}$, Victoria Neiman ${ }^{1}$, Tzippy Shochat ${ }^{3}$, \\ Aaron Sulkes ${ }^{1,2}$, Baruch Brenner ${ }^{1,2}$ and Rinat Yerushalmi ${ }^{1,2}$
}

\begin{abstract}
Background: Smoking is associated with an increased incidence of hormone receptor positive breast cancer. Data regarding worse breast cancer outcome in smokers are accumulating. Current literature regarding the impact of smoking on breast cancer characteristics is limited. We evaluated the impact of smoking on breast cancer characteristics and outcome.

Methods: This was a retrospective single center study. All women diagnosed from 4/2005 through 3/2012 and treated in our institute for early, estrogen receptor positive, human epidermal growth factor receptor 2 (HER2) negative breast cancer, whose tumors were sent for Oncotype DX analysis were included. Medical records were reviewed for demographics, clinico-pathological parameters, treatment and outcome. Data regarding smoking were retrieved according to patients' history at the first visit in the oncology clinic. Patients were grouped and compared according to smoking history (ever smokers vs. never smokers), smoking status (current vs. former and never smokers) and smoking intensity (pack years $\geq 30$ vs. the rest of the cohort). Outcomes were adjusted in multivariate analyses and included age, menopausal status, ethnicity, tumor size, nodal status and grade.

Results: A total of 662 women were included. 28.2\% had a history of smoking, 16.6\% were current smokers and 11.3\% were heavy smokers. Smoking had no impact on tumor size, nodal involvement and Oncotype DX recurrence score. Angiolymphatic and perineural invasion rates were higher in current smokers than in the rest of the cohort $(10.4 \%$ vs. $5.1 \%, p=0.045,8.3 \%$ vs. $3.5 \%, p=0.031$, respectively). Smoking had no other impact on histological characteristics. Five-year disease free survival and overall survival rates were $95.7 \%$ and $98.5 \%$, respectively. Smoking had no impact on outcomes. Adjusted disease free survival and overall survival did not influence the results.

Conclusions: Smoking had no clinically significant influence on tumor characteristics and outcome among women with estrogen receptor positive, HER2 negative, early breast cancer. As the study was limited to a specific subgroup of the breast cancer population in this heterogeneous disease and since smoking is a modifiable risk factor for the disease, further research is required to clarify the possible impact of smoking on breast cancer.
\end{abstract}

Keywords: Breast cancer, Estrogen receptor positive, Smoking, Tobacco

\section{Background}

There are accumulating data regarding the association between smoking and breast cancer. Mammary tissue is capable to uptake many tobacco carcinogens, including polycyclic aromatic hydrocarbons, aromatic amines and $\mathrm{N}$-nitrosamines. In vitro studies and animal models found that several tobacco carcinogens may induce

\footnotetext{
* Correspondence: hadar7g@gmail.com

'Institute of Oncology, Davidoff Cancer Center, Beilinson Hospital, Rabin Medical Center, 39 Jabotinski St., Petach Tikva, Israel

${ }^{2}$ Sackler Faculty of Medicine, Tel Aviv University, POB 39040, Tel Aviv, Israel

Full list of author information is available at the end of the article
}

breast tumors $[1,2]$ and may cause a more aggressive breast cancer phenotype [3]. Moreover, these carcinogens might cause DNA damage and adduct formation in mammary epithelial cells [4]. Evidence of higher prevalence of these tobacco-related DNA adducts, as well as p53 gene mutations in breast cancer tissue in smokers compared to non-smokers might implicate smoking as a factor in the pathogenesis of breast cancer [5, 6].

The existing literature links smoking with increased breast cancer incidence [7-13]. The updated International Agency for Research on Cancer (IARC) Monographs stated that smoking has a positive association with breast cancer (c) The Author(s). 2017 Open Access This article is distributed under the terms of the Creative Commons Attribution 4.0 International License (http://creativecommons.org/licenses/by/4.0/), which permits unrestricted use, distribution, and reproduction in any medium, provided you give appropriate credit to the original author(s) and the source, provide a link to the Creative Commons license, and indicate if changes were made. The Creative Commons Public Domain Dedication waiver (http://creativecommons.org/publicdomain/zero/1.0/) applies to the data made available in this article, unless otherwise stated. 
[7]. More specifically, several studies found that smoking was associated with an increased incidence of hormone receptor positive breast cancer incidence, but had no impact on triple negative breast cancer incidence [10,14].

All-cause mortality rate is higher in smokers with breast cancer compared to non-smokers [15-18]; however, the association of smoking with breast cancerspecific mortality is inconsistent [7]. Several studies found that smoking was associated with worse breast cancer specific survival (BCSS) [19-23], while others did not [24]. Some of the reports that found worse prognosis in patients with breast cancer who smoked were restricted to specific subgroups, such as heavy smokers $[25,26]$, patients with slow $\mathrm{N}$-acetyltransferase 2 activity or with tumor subtypes other than luminal B [18].

Recent meta-analysis comprising almost 40,000 patients found smoking increases risks of all-cause and breast cancer specific mortality in patients with breast cancer [23].

Smoking is a significant health problem and one of the few potentially modifiable risk factors for breast cancer development. Information regarding the impact of smoking on breast cancer characteristics is scarce. The aim of this study was to evaluate the influence of smoking on breast cancer characteristics and outcome.

We chose to focus on women with early stage, estrogen receptor (ER) positive, human epidermal growth factor receptor 2 (HER2) breast cancer as this group represent the majority of breast cancer patients [27, 28] and there is stronger association between smoking and this subgroup of patients $[10,14]$. With the addition of molecular tools, such as the Oncotype-DX (ODX) test to our daily practice $[29,30]$, patients benefit from an objective prognostic and predictive tool to evaluate risk for disease recurrence. Therefore, only women whose physician ordered the ODX test before deciding on systemic treatment were included in the study.

\section{Methods}

A retrospective single center cohort study was conducted. The study protocol was approved by the Rabin Medical Center Ethics Committee (approval number 0375-14-RMC). All women treated in our institute with ER positive, HER2 negative early breast cancer, who were diagnosed between 4/2005 and 3/2012, and whose tumors were sent for ODX analysis were included. The study cohort was generated using a registry of patients whose tumors were referred to ODX analysis and is kept in our medical center. As data were analyzed anonymously, no consent was given.

The medical records were reviewed up to $8 / 2015$ for demographics, treatment and outcome. Data regarding clinical-pathological parameters were retrieved from patients' pathological reports. Data regarding history of smoking, number of pack years and smoking status (current vs. former smoker) at breast cancer diagnosis were systematically collected according to the patients' history during the first visit at the oncology clinic. We defined current smokers as patients who actively smoked at the time of breast cancer diagnosis. Patients with history of smoking were defined as ever smoker.

Patients were grouped and compared according to their history of smoking (ever vs. never smokers), smoking status (current vs. former and never smokers), and smoking intensity ( $\geq 30$ vs. $<30$ pack years). Comparisons included tumor size, nodal involvement, stage, ODX recurrence score (RS) and histological characteristics including: ER, progesterone receptor (PR), HER2, grade, angiolymphatic and perineural invasion, Ki67 percent, P53 and histological subtype.

\section{Immunohistochemical stain reports}

ER and PR staining were reported using the modified version of the $\mathrm{H}$-score method: $1 \mathrm{x}$ percentage of weakly staining nuclei $+2 \mathrm{x}$ percentage of moderately staining nuclei $+3 \times$ percentage of intensely staining nuclei)/100, yielding a range of 0 to 3 . Ki67 staining was reported as a percentage of positively stained nuclei (0 to 100\%). HER2 negativity was defined as an immunohistochemistry (IHC) test score of 0 or 1 . If the IHC score equaled 2, negativity of HER2 was determined according to the fluorescence in situ hybridization test per the American Society of Clinical Oncology guidelines at the test time.

Survival time was defined as time from tissue diagnosis to date of last followup or death. Disease free survival (DFS) was defined as the time between surgery to event (recurrence or death) or end of followup. Duration of followup was determined according to the date of last followup in the chart. Date of recurrence was either the date of biopsy from breast or lymph node (for locoreginal recurrence) or the date of abnormal imaging (for distant recurrence). Patients' vital status was ascertained through Israel's ministry of interior database.

\section{Statistical analysis}

The statistical analysis was generated using SAS Software, Version 9.4. Continuous variables were presented by mean $\pm \mathrm{SD}$. Categorical variables were presented by $(\mathrm{N}, \%)$. T-test was used to compare the value of continuous variables between study groups. Chi-Square (for more than two groups) or Fisher's exact test (for two groups) were used to compare the value of categorical variables between study groups.

Overall survival (OS) was assessed by Kaplan-Meier survival analysis, with the log-rank test. BCSS and DFS were assessed by the Cox proportional hazards model; with the Fine and Gray correction for competing risks (the competitors were non-cancer death for BCSS and 
death without recurrence for DFS). Multivariate analysis for OS, BCSS and DFS adjusted for age, menopausal status, ethnicity and tumor characteristics including tumor size, nodal involvement and grade were assessed by the Cox proportional hazards model. Two-sided $p$-values less than 0.05 were considered statistically significant.

\section{Results}

\section{Patient characteristics}

A total of 671 patients with early breast cancer whose tumor was sent to ODX analysis were found. Nine men were excluded, remaining 662 women who were included in the study cohort. Median age was 61 years (range 34-85). 78\% women were postmenopausal. Most patients were Ashkenazi Jews (49.4\%), followed by Sephardic Jews (41.5\%), other ethnicity $(7.0 \%)$ and Arabs (2.1\%).

Data were missing regarding history of smoking in 49 (7.4\%) women, smoking status in $52(7.9 \%)$ women and number of pack years in 77 (11.6\%) women. History of smoking was present in $173(28.2 \%)$ women, and 101 (16.6\%) women were current smokers at the time of breast cancer diagnosis. 66 (11.3\%) women were heavy smokers and the median pack years was 23.5 (range 1-140). Patients characteristics and risk factors according to smoking history and status are presented in Table 1. History of smoking and smoking intensity were not associated with age, ethnicity, menopausal status, prior usage of hormone replacement therapy and family history of breast cancer. Current smokers were younger (median age 56 vs. 61, $p<0.001$ ), and were more likely to be pre-menopausal ( $p=0.002$, see Table 1$)$. Smoking status had no impact on other characteristics.
History of invasive and non-invasive breast cancer was documented in $11.2 \%$ and $2.0 \%$ of the women, respectively. Nine percent of the women had a history of malignancy other than breast cancer. Personal history of benign breast disease was noted in $9.1 \%$. Family history (first and second degree) of breast cancer and family history of any other cancer were noted in $39.7 \%$ and $58.3 \%$ of the women, respectively.

Breast cancer diagnosis was established by screening in the majority of cohort population (83.1\%). Screening rate was not associated with history of smoking or active smoking status $(p=0.363$ and $p=0.089$, respectively).

\section{Tumor characteristics}

Tumor characteristics are detailed in Tables 2 and 3 . Mean tumor size was $1.67 \mathrm{~cm}$ (SD 0.78); 83.5\% of the women had node negative disease, $7.7 \%$ had micrometastatic $(0.2-2 \mathrm{~mm})$ nodal involvement, and $8.8 \%$ had macroscopic nodal involvement. Presentation was at stage IA in $66.4 \%$, IB in $4.9 \%$, IIA in $23.2 \%$, IIB in $5.2 \%$ and IIIA in $0.3 \%$.

The most common histology was invasive ductal carcinoma (IDC) (80.8\%), followed by invasive lobular carcinoma (ILC) (12.4\%). Tumors were well, moderately and poorly differentiated in $16.7 \%, 65.0 \%$ and $18.3 \%$, respectively. Perineural and angiolymphatic invasion were noted in $4.4 \%$ and $6.0 \%$ of the women, respectively. Intensity of IHC staining of ER and PR are detailed in Table 2. As expected, there were correlations between the IHC and the oncotype ER, PR and HER2 according to real time-polymerase chain reaction (RT-PCR) results $(p<0.0001$ for all variables).

Table 1 Patients' characteristics

\begin{tabular}{|c|c|c|c|c|c|c|c|c|c|c|}
\hline \multirow[t]{2}{*}{ Population No. patients } & & \multicolumn{3}{|c|}{ Hx smoking $^{a}$} & \multicolumn{3}{|c|}{ Current Smoking $^{\mathrm{a}}$} & \multicolumn{3}{|l|}{ Pack years } \\
\hline & & Yes (173) & No (439) & $P$ value & Yes (101) & No (508) & $P$ value & $\geq 30(66)$ & $0-29(518)$ & $P$ value \\
\hline Median Age (range) & $61(34-85)$ & $60(36-83)$ & $60(34-85)$ & 0.449 & $56(36-75)$ & $61(34-85)$ & $<0.001$ & $62(43-83)$ & $60(34-85)$ & 0.197 \\
\hline \multicolumn{11}{|l|}{ Ethnicity } \\
\hline Ashkenazi & $49.5 \%$ & $46.5 \%$ & $50.1 \%$ & 0.165 & $43.9 \%$ & $50.0 \%$ & 0.449 & $52.3 \%$ & $49.5 \%$ & 0.188 \\
\hline Sephardic & $41.5 \%$ & $47.6 \%$ & $39.1 \%$ & & $49.0 \%$ & $40.1 \%$ & & $46.2 \%$ & $40.0 \%$ & \\
\hline Arab & $2.1 \%$ & $0.6 \%$ & $2.8 \%$ & & $1.0 \%$ & $2.4 \%$ & & $1.5 \%$ & $2.2 \%$ & \\
\hline Other & $7 \%$ & $5.3 \%$ & $8.0 \%$ & & $6.1 \%$ & $7.5 \%$ & & $0 \%$ & $8.3 \%$ & \\
\hline $\begin{array}{l}\text { Family } \mathrm{Hx} \text { of breast } \\
\text { cancer (yes, \%) }\end{array}$ & $39.7 \%$ & $38 \%$ & $40.3 \%$ & 0.608 & $42.4 \%$ & $39.3 \%$ & 0.564 & $38.5 \%$ & $40.4 \%$ & 0.764 \\
\hline Postmenopausal (yes, \%) & $78 \%$ & $76.7 \%$ & $77.4 \%$ & 0.914 & $64.4 \%$ & $79.7 \%$ & 0.002 & $85.1 \%$ & $76.4 \%$ & 0.122 \\
\hline Hx of HRT usage & $21.8 \%$ & $23.4 \%$ & $22.2 \%$ & 0.746 & $21 \%$ & $22.8 \%$ & 0.793 & $29.2 \%$ & $21.7 \%$ & 0.206 \\
\hline
\end{tabular}

${ }^{a}$ Data regarding smoking was retrieved during the first visit at the oncologist clinic. Current smokers were defined as patients who actively smoked at the time of breast cancer diagnosis. Patients with history of smoking were defined as ever smoker

Abbreviations: HRT hormonal replacement therapy; Hx-history

- Data were not available for: Ethnicity $(n=34)$; Family history $(n=19)$; HRT usage $(n=41)$; Hx smoking $(n=49)$; Menopausal status $(n=28)$; PY ( $n=77)$; smoking status $(n=52)$

- Cohort included patients with ER positive, HER2 negative disease 
Table 2 Tumor burden and Oncotype DX recurrence score

\begin{tabular}{|c|c|c|c|c|c|c|c|c|c|c|}
\hline \multirow[t]{2}{*}{ Population (num) } & \multicolumn{2}{|l|}{ Tumor size } & \multicolumn{2}{|c|}{ Macroscopic node positive ${ }^{a}$} & \multicolumn{4}{|c|}{ Stage (\%) } & \multicolumn{2}{|c|}{ Oncotype Dx RS } \\
\hline & Mean cm (SD) & $P$ & $(\%)$ & $P$ & I & $\|$ & III & $P$ & Mean (SD) & $P$ \\
\hline All (662) & $1.67(0.78)$ & - & 8.8 & - & 71.3 & 28.4 & 0.3 & - & $19.03(10.23)$ & - \\
\hline \multicolumn{11}{|l|}{ Hx smoking ${ }^{b}$} \\
\hline Yes (173) & $1.66(0.87)$ & 0.642 & 9.9 & 0.574 & 73.8 & 25.6 & 0.6 & 0.695 & $18.69(9.15)$ & 0.447 \\
\hline No (439) & $1.69(0.75)$ & & 8.5 & & 69.4 & 30.4 & 0.2 & & $19.35(10.78)$ & \\
\hline \multicolumn{11}{|l|}{ Current smoking $^{\mathrm{b}}$} \\
\hline Yes (101) & $1.76(0.98)$ & 0.392 & 8 & 0.73 & 73.0 & 26.0 & 1.0 & 0.613 & $19.42(8.52)$ & 0.70 \\
\hline No (508) & $1.67(0.74)$ & & 9.1 & & 70.2 & 29.6 & 0.2 & & $19.05(10.49)$ & \\
\hline \multicolumn{11}{|c|}{ Number of pack years (PY) } \\
\hline $\mathrm{PY}^{2} 0-29(518)$ & $1.68(0.76)$ & 0.82 & 9.5 & 0.38 & 69 & 30.6 & 0.4 & 0.172 & $19.25(10.66)$ & 0.454 \\
\hline PY $>=30$ (66) & $1.65(0.96)$ & & 6.2 & & 80.0 & 20.0 & 0 & & $18.37(8.68)$ & \\
\hline
\end{tabular}

${ }^{\mathrm{a}}$ Macroscopic nodes- lymph node metastases $>2 \mathrm{~mm}$

${ }^{b}$ Data regarding smoking was retrieved during the first visit at the oncologist clinic. Current smokers were defined as patients who actively smoked at the time of breast cancer diagnosis. Patients with history of smoking were defined as ever smoker

- Abbreviations: $\mathrm{cm}$ centimeter, $\mathrm{Hx}$ history, $P Y$ pack years, $R S$ recurrence score, $S D$ standard deviation

- Data were not available for: Hx smoking $(n=49)$; nodal status $(n=2)$; PY $(n=77)$; smoking status $(n=52)$; Stage $(n=2)$; Tumor size $(n=1)$

- Cohort included patients with ER positive, HER2 negative disease

Median ODX score was 17 (range 0-88) with 50.6\%, $38.3 \%$ and $11.1 \%$ of the patients had low (0-17), intermediate (18-30) and high ( $\geq 31)$ RS, respectively.

\section{Smoking and tumor characteristics}

Tumor characteristics according to the evaluated groups are depicted in Tables 2 and 3. Smoking history, smoking status and number of pack years smoked were not associated with tumor and histological characteristics, except with angiolymphatic and perineural invasion, which were higher in current smokers $(10.4 \%$ vs. $5.1 \%$, $p=0.045,8.3 \%$ vs. $3.5 \%, p=0.031$, respectively).

\section{Treatment}

Adjuvant hormonal treatment was administered to $97.7 \%$ women, most commonly starting with tamoxifen. Most women (74.4\%) did not receive adjuvant chemotherapy. Adjuvant treatment with docetaxel and cyclophosphamide was administered to $11 \%$, anthracycline based chemotherapy without taxane was administered to $9.4 \%$, a combination of anthracycline with cyclophosphamide followed by a taxane to $3.7 \%$ and other combinations to $1.5 \%$ of the women. History of smoking and active smoking were not associated with adjuvant hormonal or chemotherapy treatment.

\section{Outcome}

Median follow up was 61.9 months (range 1.7-114.6). During this period, $13(2 \%)$ women died of breast cancer, $14(2.1 \%)$ died of other causes and 635 (95.9\%) remained alive. Outcome according to smoking groups are depicted in Table 4 . Breast cancer recurrence rate was $5.1 \%$ for the study cohort. Recurrence rates were $3.6 \%, 5.3 \%$ and $11 \%$ for patients with low, intermediate and high RS, respectively $(p=0.036)$. Accordingly, the estimated 5-year DFS rates for all patients were 95.7\% and $96.6 \%$ for patients with low RS, 95.6\% for intermediate RS and $91.8 \%$ for high RS. Five-year DFS rate was significantly different between patients with low and high RS (hazard ratio $=0.40,95 \%$ CI $0.16-0.98$, $p=0.045)$. History of smoking and smoking status were not associated with recurrence rate $(p=0.424$ and $p=0.128$, respectively). DFS rates were not associated with history of smoking ( $\mathrm{HR}=0.73,95 \% \mathrm{CI} 0.32-1.68$, $p=0.456)$, smoking status ( $\mathrm{HR}=0.36,95 \%$ CI 0.09 $1.48, p=0.155)$ and number of pack years $(\mathrm{HR}=0.85$, 95\% CI 0.26-2.80, $p=0.794)$.

Five-year OS rate was $98.5 \%$. OS rates were not associated with history of smoking ( $\mathrm{HR}=0.80,95 \% \mathrm{CI} 0.22$ $2.89, p=0.742)$, and number of pack years $(\mathrm{HR}=1.50$, 95\% CI 0.33-6.78, $p=0.596)$. Calculation of HR for OS according to smoking status was not applicable. Fiveyear BCSS was $98.5 \%$. BCSS rates were not associated with smoking status $(\mathrm{HR}=0.53,95 \% \mathrm{CI} 0.12-2.38$, $p=0.404)$ and number of pack years $(\mathrm{HR}=0.73,95 \%$ CI 0.09-5.70, $p=0.764$ ). Calculation of HR for BCSS according to smoking status was not applicable.

Adjustment of HRs for DFS, OS and BCSS for age, menopausal status, ethnicity, tumor size, nodal involvement and grade were similar for history of smoking, smoking status and number of pack years (see Table 5).

\section{Discussion}

We evaluated the associations between smoking and ER positive, HER2 negative early breast cancer characteristics and outcome. Current smokers had significantly higher angiolymphatic $(n=11)$ and perineural invasion $(n=9)$ compared to the rest of the study cohort. However, due to the low numbers of patients with these 


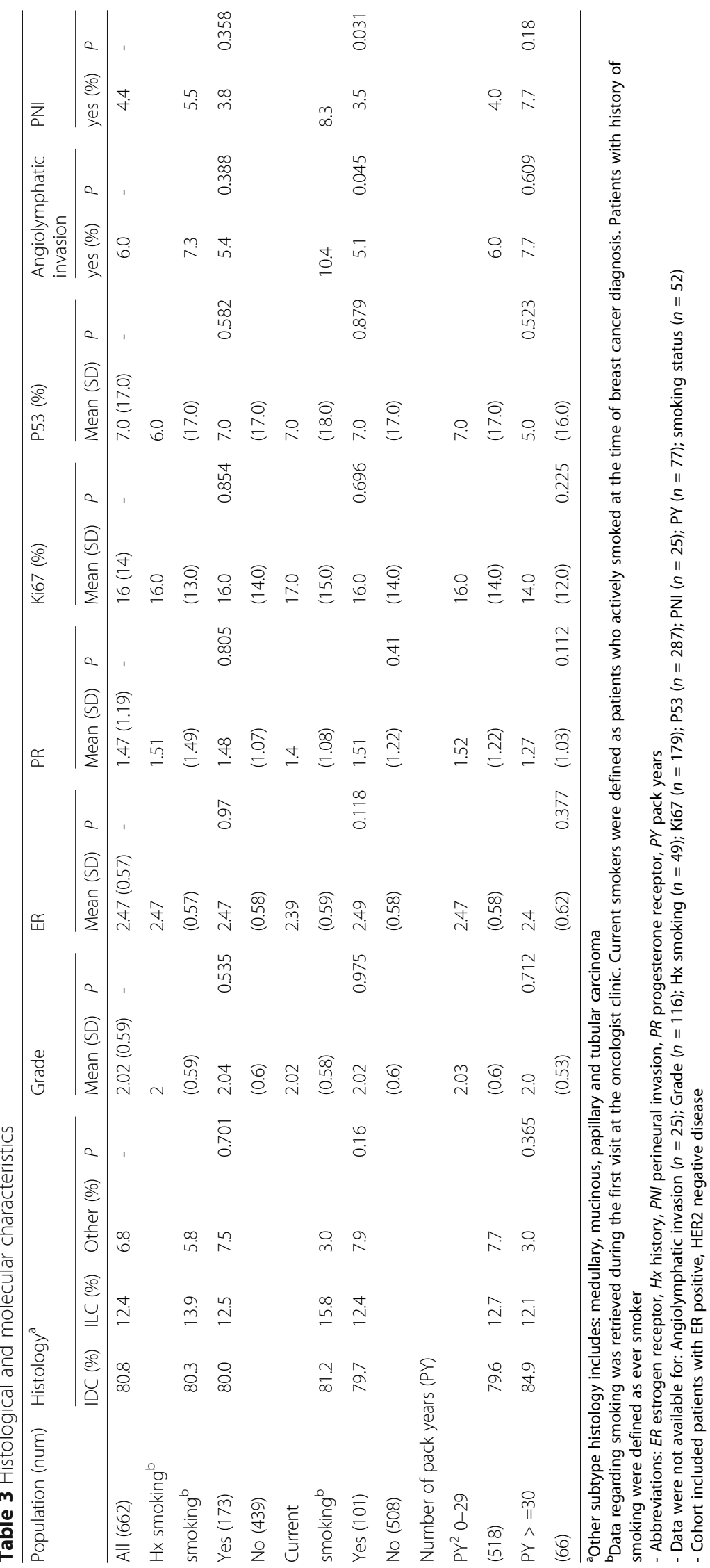


Table 4 Five-year disease free survival, breast cancer specific survival and overall survival

\begin{tabular}{|c|c|c|c|c|c|c|c|c|c|}
\hline \multirow[t]{2}{*}{ Population (num) } & \multicolumn{3}{|l|}{ DFS } & \multicolumn{3}{|l|}{ BCSS } & \multicolumn{3}{|l|}{ OS } \\
\hline & 5-y DFS & $\mathrm{HR} 95 \% \mathrm{Cl}$ & $p$ & 5-y BCSS & $\mathrm{HR} 95 \% \mathrm{Cl}$ & $p$ & 5 -y OS & $\mathrm{HR} 95 \% \mathrm{Cl}$ & $p$ \\
\hline All (662) & $95.7 \%$ & - & - & $95.8 \%$ & - & - & $98.5 \%$ & - & - \\
\hline \multicolumn{10}{|l|}{ Hx smoking ${ }^{b}$} \\
\hline Yes (173) & $96.4 \%$ & 0.73 & 0.456 & $99.2 \%$ & 0.53 & 0.404 & $98.8 \%$ & 0.80 & 0.74 \\
\hline No (439) & $95.1 \%$ & $(0.32-1.68)$ & & $98.4 \%$ & $(0.12-2.38)$ & & $98.6 \%$ & $(0.22-2.92)$ & \\
\hline \multicolumn{10}{|l|}{ Current Smokering ${ }^{\mathrm{b}}$} \\
\hline Yes (101) & $98.2 \%$ & 0.36 & 0.155 & NA & NA & NA & NA & NA & NA \\
\hline No (508) & $95.1 \%$ & $(0.09-1.48)$ & & & & & & & \\
\hline \multicolumn{10}{|c|}{ Number of pack years (PY) } \\
\hline$P Y^{2} 0-29(518)$ & $95.2 \%$ & 0.85 & 0.794 & $98.5 \%$ & 0.73 & & $98.6 \%$ & 1.50 & 0.596 \\
\hline PY > =30 (66) & $96.1 \%$ & $(0.26-2.80)$ & & $99.1 \%$ & $(0.09-5.70)$ & 0.764 & $98.5 \%$ & $(0.33-6.78)$ & \\
\hline
\end{tabular}

${ }^{a}$ Sample size: DFS: Smoking history $n=599$, smoking status $n=596$, pack years $n=575$, BCSS: Smoking history $n=607$, smoking status $n=604$, pack years $n=580$, OS: Smoking history $n=610$, smoking status $n=607$, pack years $n=582$

${ }^{b}$ Data regarding smoking was retrieved during the first visit at the oncologist clinic. Current smokers were defined as patients who actively smoked at the time of breast cancer diagnosis. Patients with history of smoking were defined as ever smoker

- Abbreviations: BCSS breast cancer specific survival, DFS disease free survival, NA not applicable, OS overall survival

- Cohort included patients with ER positive, HER2 negative disease

histopathological characteristics, the analysis for this population is limited. Tumor burden, ODX RS and other histological characteristics were not associated with smoking. Lack of differences in traditional prognostic factors such as tumor size, nodal involvement, intensity of IHC stain, grade and Ki67 percent are in line with similar ODX RS in all groups.

Some reports suggested that smoking is associated with increased breast cancer incidence [7-13] and worse outcome [15-23, 31]. However, the magnitude and mechanism of the impact of tobacco on breast cancer development remains unclear, and data regarding the association of smoking and breast cancer characteristics are scarce.

As a result of the decline in smoking prevalence in recent decades observed in many developed countries, former smokers tend to outnumber current smokers [32, 33]. However, in our study the number of current smoker was higher than the number of former smokers (101 vs. 72). This discordance may raise concern for potential bias. A possible explanation could be the inclusion of women whose tumor was sent to ODX analysis. Women who are candidates for adjuvant chemotherapy tend to be younger than the "average" breast cancer population. Indeed, a recent large study evaluating the association between smoking status and breast cancer showed that the number of current smokers might actually outnumber the number of former smokers [35].

This study did not support previous reports showing worse outcome among patients with breast cancer and history of smoking. Both adjusted and unadjusted

Table 5 Adjusted Five-year disease free survival, breast cancer specific survival and overall survival ${ }^{a}$

\begin{tabular}{|c|c|c|c|c|c|c|}
\hline Population ${ }^{\text {b }}$ (num) & 5-y DFS HR 95\% Cl & $p$ & 5-y BCSS HR 95\% Cl & $p$ & 5-y OS HR 95\% Cl & $p$ \\
\hline \multicolumn{7}{|l|}{ Hx smoking ${ }^{c}$} \\
\hline Yes (178) & 0.65 & 0.418 & 0.66 & 0.632 & 1.14 & 0.852 \\
\hline No (443) & $(0.23-1.83)$ & & $(0.12-3.58)$ & & $(0.28-4.71)$ & \\
\hline \multicolumn{7}{|l|}{ Current Smoking $^{3}$} \\
\hline Yes (104) & 0.46 & 0.33 & NA & NA & NA & NA \\
\hline No (513) & $(0.10-2.18)$ & & & & & \\
\hline \multicolumn{7}{|c|}{ Number of pack years (PY) } \\
\hline $\mathrm{PY}^{3} 0-29$ (523) & 0.69 & 0.616 & 1.02 & 0.984 & 2.15 & 0.344 \\
\hline PY $>=30(67)$ & $(0.16-2.96)$ & & $(0.12-8.40)$ & & $(0.44-10.49)$ & \\
\hline
\end{tabular}

${ }^{a}$ Multivariate adjustment included the following covariates: age, menopausal status, ethnicity, tumor size, nodal involvement and grade

${ }^{b}$ Sample size: DFS: Smoking history $n=439$, smoking status $n=437$, pack years $n=423$, BCSS: Smoking history $n=443$, smoking status $n=441$, pack years $n=425$, OS: Smoking history $n=444$ smoking status $n=442$, pack years $n=426$

'Data regarding smoking was retrieved during the first visit at the oncologist clinic. Current smokers were defined as patients who actively smoked at the time of breast cancer diagnosis. Patients with history of smoking were defined as ever smoker

- Abbreviations: BCSS breast cancer specific survival, DFS disease free survival, NA not applicable, OS overall survival

- Cohort included patients with ER positive, HER2 negative disease 
analyses of DFS, BCSS and OS showed smoking was not associated with different outcome. However, as only 27 deaths occurred during the followup period, and only 13 deaths were related to breast cancer, conclusions that can be drawn regarding the impact of smoking on outcome in this cohort are limited. Given the excellent outcome for patients with ER positive early breast cancer, a larger population and longer follow-up are required to identify any possible differences.

This study was confined to a specific sub-group of breast cancer patients. In addition, smoking might be associated with molecular and genetic changes which are not expressed by the tumor characteristics collected for this analysis. Therefore, whether smoking has any impact on breast cancer biology remains to be determined.

The study has several limitations. First, as this is a single center, retrospective study, it is vulnerable to unknown bias. Data regarding body mass index (BMI) and physical activity, which have been shown to be associated with breast cancer, were limited and therefore were not adjusted for. Second, smoking history was acquired during the first visit at the oncology clinic and is subjective to recall and reporting biases. Moreover, information regarding smoking status during the followup period was missing, which presents a limitation for outcome analysis according to smoking status. However, we believe the women who were included in this study were unlikely to start smoking given a median age of 61 years. Furthermore, the change in number of heavy smokers during the median followup of 5 years is also probably insignificant. Third, passive smoking, which might also have impact on breast cancer [11,34], was not recorded. Moreover, age at smoking initiation and history of pack years smoked before first pregnancy were not recorded. Since several reports support the correlation between number of pack years before first pregnancy and breast cancer risk [35, 36], these data might help us understand the influence of smoking on breast cancer better.

Strengths of this study include the large patient cohort. Furthermore, the chart review included detailed, individual patient data, lacking in registry-based studies. The correlation between IHC staining and the ODX ER, PR and HER2 (according to RT-PCR) results, as well as the correlation between ODX RS and the DFS of the study population add to the reliability and validity of the findings.

\section{Conclusions}

In this study cohort, smoking had no clinically significant impact on tumor characteristics and outcome among women with ER positive, HER2 negative early breast cancer. Of note, breast cancer is a heterogeneous disease and these results are limited to a specific subgroup of patients. As smoking is a common health problem and an established modifiable risk factor for breast cancer development, further studies are needed to elucidate the possible impact of smoking on breast cancer patients.

\section{Abbreviations \\ BCSS: Breast cancer specific survival; DFS: Disease free survival; ER: Estrogen receptor; HER2: Human epidermal growth factor receptor 2; IDC: Invasive ductal carcinoma; IHC: Immunohistochemistry; ILC: Invasive lobular carcinoma; \\ ODX: Oncotype-DX; OS: Overall survival; PR: Progesterone receptor; \\ RS: Recurrence score; RT-PCR: Real time-polymerase chain reaction}

\section{Acknowledgments}

The authors would like to thank Ms. Faye Schrieber for providing professional writing service.

\section{Funding}

None- for all authors.

\section{Availability of data and materials}

Data supporting our findings are presented in the "Results" section. Researchers interested in source data are invited to write to the corresponding author

\section{Authors' contributions}

HG: Substantial contributions to study conception and design, acquisition of data, analysis and interpretation of data, drafting the article, revising it critically for important intellectual content, final approval of the version to be published, agree to be accountable for all aspects of the work. OG: Substantial contributions to study conception and design, analysis and interpretation of data, revising it critically for important intellectual content, final approval of the version to be published, agree to be accountable for all aspects of the work. SR: Substantial contributions to study conception and design, analysis and interpretation of data, revising the article critically for important intellectual content, final approval of the version to be published, agree to be accountable for all aspects of the work. DH: Substantial contributions to acquisition of data, revising the article critically for important intellectual content, final approval of the version to be published, agree to be accountable for all aspects of the work, agree to be accountable for all aspects of the work. VN: Substantial contributions to acquisition of data, revising the article critically for important intellectual content, final approval of the version to be published.

TS: Substantial contributions to analysis and interpretation of data, revising the article critically for important intellectual content, final approval of the version to be published, agree to be accountable for all aspects of the work. AS: Substantial contributions to analysis and interpretation of data, revising the article critically for important intellectual content, final approval of the version to be published, agree to be accountable for all aspects of the work. BB: Substantial contributions to analysis and interpretation of data, revising the article critically for important intellectual content, final approval of the version to be published, agree to be accountable for all aspects of the work. RY: Substantial contributions to study conception and design, analysis and interpretation of data, drafting the article, revising it critically for important intellectual content, final approval of the version to be published, agree to be accountable for all aspects of the work.

\section{Ethics approval and consent to participate}

The study protocol was approved by the Rabin Medical center Ethics Committee (approval number 0375-14-RMC). As data were analyzed anonymously, no consent was given.

Consent for publication

Not applicable.

\section{Competing interests}

The authors declare that they have no financial and non-financial competing interests. 


\section{Publisher's Note}

Springer Nature remains neutral with regard to jurisdictional claims in published maps and institutional affiliations.

\section{Author details}

'Institute of Oncology, Davidoff Cancer Center, Beilinson Hospital, Rabin Medical Center, 39 Jabotinski St., Petach Tikva, Israel. ${ }^{2}$ Sackler Faculty of Medicine, Tel Aviv University, POB 39040, Tel Aviv, Israel. ${ }^{3}$ Statistical Consulting Unit, Beilinson Hospital, Rabin Medical Center, 39 Jabotinski St., Petach Tikva, Israel.

Received: 1 March 2016 Accepted: 23 August 2017

Published online: 06 September 2017

\section{References}

1. Terry PD, Goodman MI. The association between cigarette smoking and breast cancer modified by genotype? A review of epidemiologic studies and meta-analysis. Cancer Epidemiol Biomark Prev. 2006;15:602-11.

2. Reynolds P (2013) Smoking and breast cancer. J mammary gland biol Neoplasia. 2013;18:15-23

3. Di Cello F, Flowers VL, Li H, Vecchio-Pagán B, Gordon B, Harbom K, et al. Cigarette smoke induces epithelial to mesenchymal transition and increases the metastaticability of breast cancer cells. Mol Cancer. 2013;12:90.

4. Catsburg C, Kirsh VA, Soskolne CL, Kreiger N, Rohan TE. Active cigarette smoking and the risk of breast cancer: a cohort study. Cancer Epidemiol. 2014;38:376-81.

5. Conway K, Edmiston SN, Cui L, Drouin SS, Pang J, He M, et al. Prevalence and spectrum of p53 mutations associated with smoking in breast cancer. Cancer Res. 2002;62:1987-95.

6. Li D, Zhang W, Sahin AA, Hittelman WNDNA. Adducts in normal tissue adjacent to breast cancer: a review. Cancer Detect Prev. 1999;23:454-62.

7. International Agency for Research on Cancer. IARC monographs on the evaluation of carcinogenic risks to humans tobacco smoking, vol. 100E. Lyon, France: IARC Press; 2012. p. 92-101.

8. Alberg AJ, Shopland DR, Cummings KM. The 2014 surgeon general's report: commemorating the $50^{\text {th }}$ anniversary of the 1964 report of the advisory committee to the US surgeon general and updating the evidence on the health consequences of cigarette smoking. Am J Epidemiol. 2014;179:403-12.

9. Johnson KC, Miller AB, Collishaw NE, Palmer JR, Hammond SK, Salmon AG, et al. Active smoking and secondhand smoke increase breast cancer risk: the report of the Canadian expert panel on tobacco smoke and breast cancer risk (2009). Tob Control. 2011;20:e2.

10. Kawai M, Malone KE, Tang MC. Active smoking and the risk of estrogen receptor-positive and triple-negative breast cancer among women ages 20 to 44 years. Cancer. 2014;120:1026-34.

11. Rosenberg $L$, Boggs DA, Bethea $T N$, Wise LA, Adams-Campbell LL, Palmer JR. A prospective study of smoking and breast cancer risk among African American wome. Cancer Causes Control. 2013;24:2207-15.

12. Band PR, Le ND, Fang R, Deschamps M. Carcinogenic and endocrine disrupting effects of cigarette smoke and risk of breast cancer. Lancet. 2002 360:1044-9.

13. Macacu A, Autier P, Boniol M, Boyle P. Active and passive smoking and risk of breast cancer: a meta-analysis. Breast Cancer Res Treat. 2015;154:213-24.

14. Kabat GC, Kim M, Phipps Al, Li Cl, Messina CR, Wactawski-Wende J, et al. Smoking and alcohol consumption in relation to risk of triple-negative breast cancer in a cohort of postmenopausal women. Cancer Causes Control. 2011;22:775-83.

15. Holmes MD, Murin S, Chen WY, Kroenke CH, Spiegelman D, Colditz GA. Smoking and survival after breast cancer diagnosis. Int J Cancer. 2007;20:2672-7.

16. Tominaga K, Andow J, Koyama Y, Numao S, Kurokawa E, Ojima M, et al. Family environment, hobbies and habits as psychosocial predictors of survival for surgically treated patients with breast cancer. Jpn J Clin Oncol. 1998:28:36-41.

17. Hellmann SS, Thygesen LC, Tolstrup JS, Grønbaek M. Modifiable risk factors and survival in women diagnosed with primary breast cancer: results from a prospective cohort study. Eur J Cancer Prev. 2010;19:366-73.

18. Seibold P, Vrieling A, Heinz J, Obi N, Sinn HP, Flesch-Janys D, et al. Prediagnostic smoking behaviour and poorer prognosis $n$ a German breast cancer patient cohort- differential effects by tumour subtype, NAT2 status, BMI and alcohol intake. Cancer Epidimiol. 2014;38:419-26.
19. Braithwaite D, Izano M, Moore DH, Kwan ML, Tammemagi MC, Hiatt RA, et al. Smoking and survival after breast cancer diagnosis: a prospective observational study and systematic review. Breast Cancer Res Treat. 2012:136:521-33.

20. Fentiman IS, Allen DS, Hamed H. Smoking and prognosis in women with breast cancer. Int J Clin Pract. 2005:59:1051-4.

21. Boone SD, Baumgartner KB, Baumgartner RN, Connor AE, John EM, Giuliano $A R$, et al. Active and passive cigarette smoking and mortality among Hispanic and non-Hispanic white women diagnosed with invasive breast cancer. Ann Epidemiol. 2015;25:824-31.

22. Bérubé S, Lemieux J, Moore L, Maunsell E, Brisson J. Smoking at time of diagnosis and breast cancer-specific survival: new findings and systematic review with meta-analysis. Breast Cancer Res. 2014;16:R42.

23. Wang K, Li F, Zhang X, Li Z, Li H. Smoking increases risks of all-cause and breast cancer specific mortality in breast cancer individuals: a dose-response meta-analysis of prospective cohort studies involving 39725 breast cancer cases. Oncotarget. 2016;7:83134-47.

24. Sagiv SK, Gaudet MM, Eng SM, Abrahamson PE, Shantakumar S, Teitelbaum SL, et al. Active and passive cigarette smoke and breast cancer survival. Ann Epidemiol. 2007;17:385-93.

25. Pierce JP, Patterson RE, Senger CM, Flatt SW, Caan BJ, Natarajan L, et al. Lifetime cigarette smoking and breast cancer prognosis in the after breast cancer pooling project. J Natl Cancer Inst. 2014;106:djt359.

26. Saquib N, Stefanick ML, Natarajan L, Pierce JP. Mortality risk in former smokers with breast cancer: pack-years vs. smoking status. Int J Cancer. 2013:133:2493-7.

27. Early Breast Cancer Trialists' Collaborative Group (EBCTCG), Davies C, Godwin J, Gray R, Clarke M, Cutter D, Darby S, et al. Relevance of breast cancer hormone receptors and other factors to the efficacy of adjuvant tamoxifen: patient-level meta-analysis of randomised trials. Lancet. 2011;378:771-84.

28. Harvey JM, Clark GM, Osborne CK, Allred DC. Estrogen receptor status by immunohistochemistry is superior to the ligand-binding assay for predicting response to adjuvant endocrine therapy in breast cancer. J Clin Oncol. 1999; 17:1474-81

29. Paik S, Tang G, Shak S, Kim C, Baker J, Kim W, et al. Gene expression and benefit of chemotherapy in women with node-negative, estrogen receptorpositive breast cancer. J Clin Oncol. 2006;24:3726-34.

30. Paik S, Shak S, Tang G, Kim C, Baker J, Cronin M, et al. A multigene assay to predict recurrence of tamoxifen-treated, node-negative breast cancer. New Engl J Med. 2004;351:2817-26.

31. Bishop JD, Killelea BK, Chagpar AB, Horowitz NR, Lannin DR. Smoking and breast cancer recurrence after breast conservation therapy. Int J Breast Cancer. 2014; https://doi.org/10.1155/2014/327081.

32. Passarelli MN, Newcomb PA, Hampton JM, Trentham-Dietz A, Titus LJ, Egan KM, et al. Cigarette smoking before and after breast cancer diagnosis: mortality from breast cancer and smoking-related diseases. J Clin Oncol. 2016:34:1315-22.

33. Https://www.cdc.gov/tobacco/data_statistics/fact_sheets/adult_data/cig_ smoking/

34. Chen C, Huang YB, Liu XO, Gao Y, Dai HJ, Song FJ, et al. Active and passive smoking with breast cancer risk for Chinese females: a systematic review and meta-analysis. Chin J Cancer. 2014;33:306-16.

35. Gaudet MM, Gapstur SM, Sun J, Diver WR, Hannan LM, Thun MJ. Active smoking and breast cancer risk: original cohort data and meta-analysis. J Natl Cancer Inst. 2013;105:515-25.

36. Xue F, Willett WC, Rosner BA, Hankinson SE, Michels KB. Cigarette smoking and the incidence of breast cancer. Arch Intern Med. 2011;171:125-33. 